\title{
Diagnostic Accuracy and Complication Rates of Fusion Images Created Using Real-Time Ultrasound with CT for Identification of Peripheral Lung Lesions in Patients Undergoing Biopsy
}

\author{
Rinpei Imamine ${ }^{1,2}$, Hisato Kobayashi'2, Keizo Akuta ${ }^{2}$, Mitsuru Matsuki ${ }^{3}$, Hiroyoshi Isoda4, \\ Kaori Togashi ${ }^{1}$
}

${ }^{1}$ Department of Radiology, Kyoto University Graduate School of Medicine, Kyoto, Japan

${ }^{2}$ Department of Radiology, Japanese Red Cross Otsu Hospital, Otsu-shi, Japan

${ }^{3}$ Department of Radiology, Kinki University School of Medicine, Sayama-City, Japan

${ }^{4}$ Preemptive Medicine and Lifestyle-Related Disease Research Center, Kyoto University Hospital, Kyoto, Japan

Email: imamine.rinpei.2n@kyoto-u.jp

How to cite this paper: Imamine, R., Kobayashi, H., Akuta, K., Matsuki, M., Isoda, H. and Togashi, K. (2019) Diagnostic Accuracy and Complication Rates of Fusion Images Created Using Real-Time Ultrasound with CT for Identification of Peripheral Lung Lesions in Patients Undergoing Biopsy. Open Journal of Radiology, 9, 36-47.

https://doi.org/10.4236/ojrad.2019.91004

Received: January 8, 2019

Accepted: January 22, 2019

Published: January 25, 2019

Copyright $\odot 2019$ by author(s) and Scientific Research Publishing Inc. This work is licensed under the Creative Commons Attribution International License (CC BY 4.0).

http://creativecommons.org/licenses/by/4.0/

\begin{abstract}
Background: Fusion image improves lesion detectability and can be an effective tool for percutaneous ultrasound (US)-guide procedure. We describe the clinical benefit of US-guided lung biopsy using fusion image. Purpose: To retrospectively compare the diagnostic accuracy and complication rates of US-guided lung biopsy with B-mode alone and those of a fusion image created using real-time US and computed tomography (CT). Materials and Methods. Between September, 2013 and September, 2016, 50 peripheral lung lesions in 50 patients ( 40 males, 10 females; median, 74 years old) were performed by US-guided percutaneous cutting needle biopsy using the B-mode alone or fusion image. Final diagnoses were based on surgical outcomes or clinical follow-up results for at least 12 months after biopsy. To assess prebiopsy characteristics, all lesions were divided into two groups: group 1 (identification on B-mode) and group 2 (identification on fusion image). Results. Of 50 peripheral lesions, 40 lesions (80\%) were detected by means of B-mode alone (group 1), and 10 lesions (20\%) were identified by fusion image (group 2). The diagnostic accuracy of group 1 was $90 \%$ (36/40 lesions), and the diagnostic accuracy of group 2 was $100 \%$ (10/10 lesions). Nodule type and the size of the lesions showed significant group wise differences $(p<0.001$ and $p=0.02$, respectively). Pneumothorax occurred immediately after the first
\end{abstract}


puncture in five of $50(10 \%)$ lesions, with no symptom development in all patients. Conclusion: Fusion images created using real-time US and CT may be useful for identification of the minimal size of potential target lung lesions and may be more suitable for improved yields with US-guided lung biopsy.

\section{Keywords}

Ultrasound, Fusion Image, US-Guided Lung Biopsy, Diagnostic Accuracy, Safety

\section{Introduction}

In the era of personalized medicine in oncology, the requirement for a least invasive biopsy technique that can provide accurate diagnosis is becoming increasingly important. Several investigators have described the utility and safety of ultrasound (US)-guided transthoracic cutting needle biopsy and fine needle aspiration in chest lesions [1]-[10]. However, these studies were conducted only in the conventional B mode, and thus occurrence of mis-targeting is possible. Although careful US examination is required to detect the contact area between the peripheral lung lesion and the pleura, conditions such as pleural adhesions and bullous emphysema may interfere with US-visualization of the pleural surface [11] [12].

Recently, fusion image with real-time US and computed tomography (CT) has been introduced in interventional radiology [13] [14]. In the chest, fusion image can be used to assist in detecting the pleural-lesion contact area in extremely small solid nodules. Moreover, the fusion image of the pleural membrane associated with solid component of part-solid ground-glass nodule (GGN), results in localization of the lesion. The effectiveness of fusion image for US-guided lung biopsy has not been reported. The purpose of this study was to compare the diagnostic accuracy and complication rates associated with US-guided lung biopsy with B-mode alone and those of fusion images created using real-time US and CT.

\section{Materials and Methods}

\subsection{Patients}

The institutional review board approved this retrospective study and waived the requirement for informed consent. This retrospective study was conducted at a single institution. Between September, 2013 and September, 2016, US-guided percutaneous cutting needle biopsy was conducted for 63 thoracic lesions. Biopsy was performed for all patients within 4 weeks after a chest lesion was detected with CT. Of these, 12 mediastinal lesions and one lung lesion were excluded because of the use of contrast-enhanced US for detection of the lesion [15]. The 
remaining 50 peripheral lesions in 50 patients (40 males and 10 females; age range, 50 - 86 years old; median age, 74 years old) were examined in this study.

\subsection{US Examination and US-Guided Biopsy Procedure}

All patients were evaluated using the GE LOGIQ Q9 US imaging system (GE Healthcare Worldwide, Chicago, IL, USA). US examinations were performed by an interventional radiologist (with experience of $>30$ years). Patients were placed in the supine, prone, or right/left lateral decubitus positions. The indication of fusion image depended on the same interventional radiologist. Initially, US using the conventional B-mode was conducted to identify CT-detected lesions. When a solid nodule was not identified through conventional B-mode, fusion image with real-time US and CT was performed using a commercially available US unit (Figure 1). Moreover, fusion image was successful in localizing the part-solid GGN with pleural involvement, despite non-detection of the ground-glass opacity within the sonographic window (Figure 2). For the fusion image, the manubrium of the sternum served as a reference point to synchronize the spatial relationship between the CT and US images in supine position. In the prone or lateral position, the first or $12^{\text {th }}$ spinous process was used as a reference point. Once

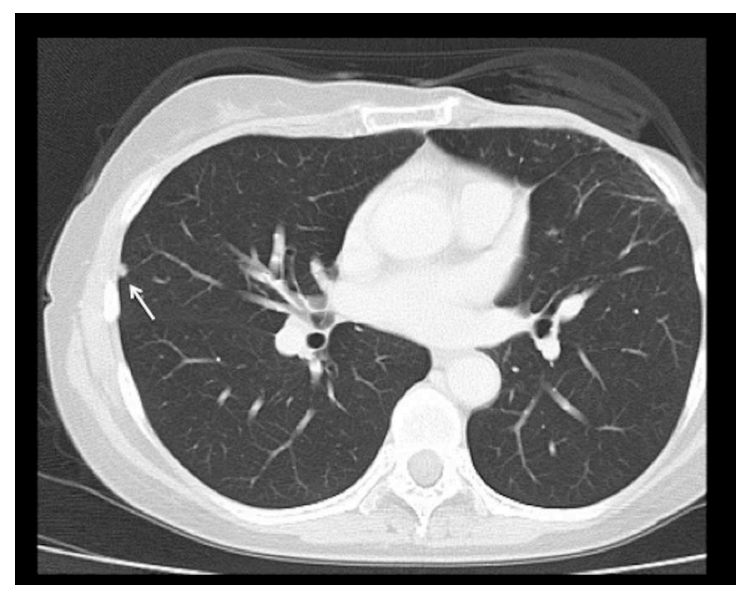

(a)

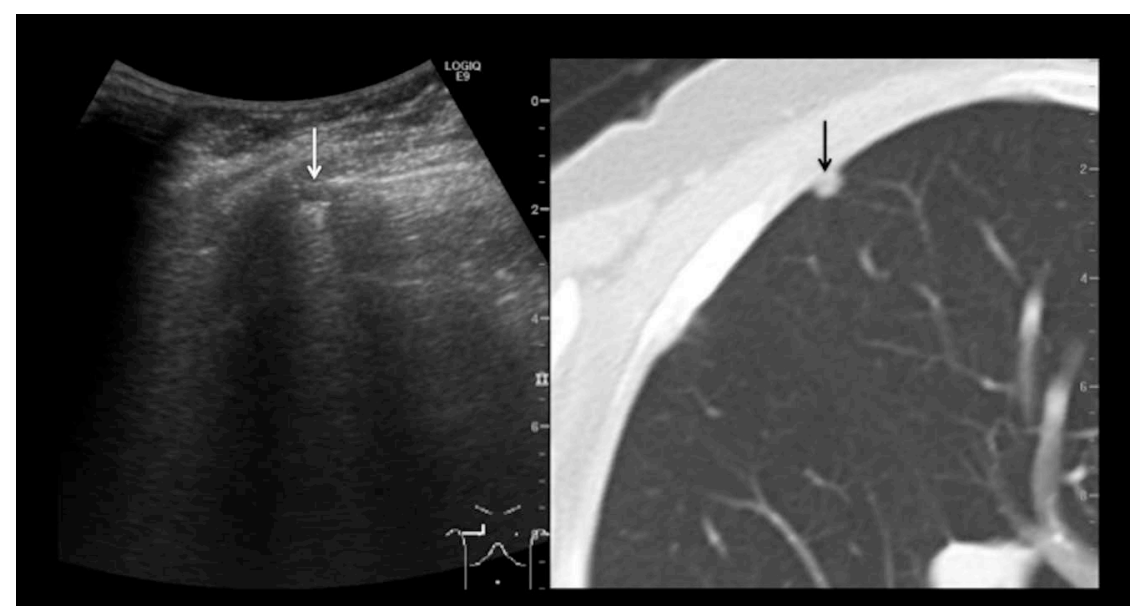

(b) 


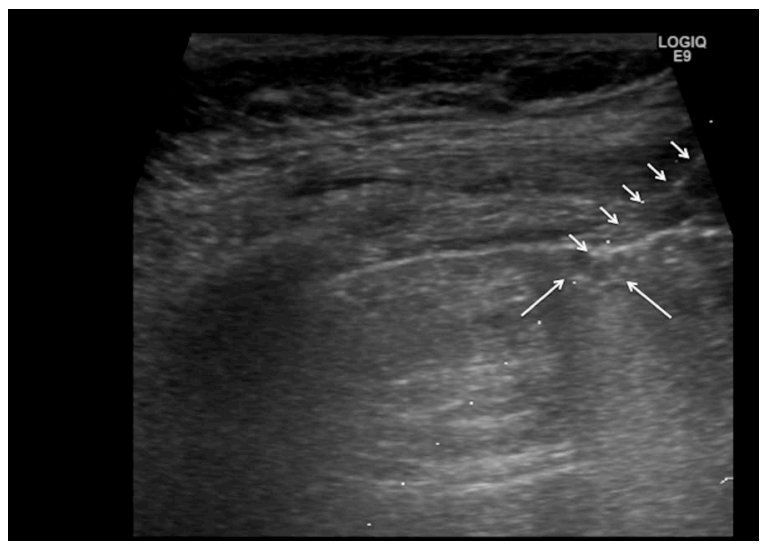

(c)

Figure 1. Pulmonary nodule in a 61-year-old woman with a history of minor salivary gland tumor (adenoid cystic carcinoma) and breast cancer. (a) Chest CT scan showed a 4-mm peripheral solid nodule (arrow) in the right middle lobe; (b) Although B-mode alone failed to reveal the solid nodule, localization of the solid nodule was possible using fusion image with real-time US (white arrow) and CT (black arrow); (c) The inserted line was visualized as a dotted line; subsequently, the biopsy needle (short arrows) was introduced into the lesion (long arrows) along the dotted line. Only one puncture was performed due to pneumothorax. The biopsy revealed metastatic lesion of minor salivary gland tumor (adenoid cystic carcinoma).

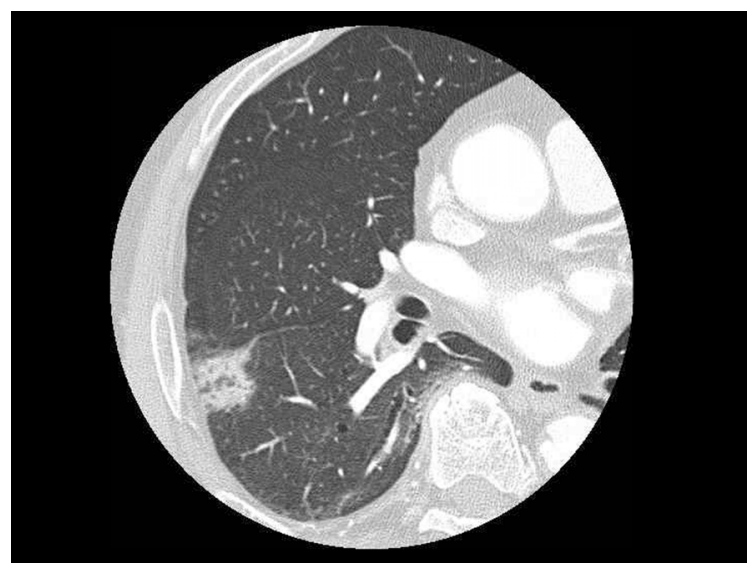

(a)

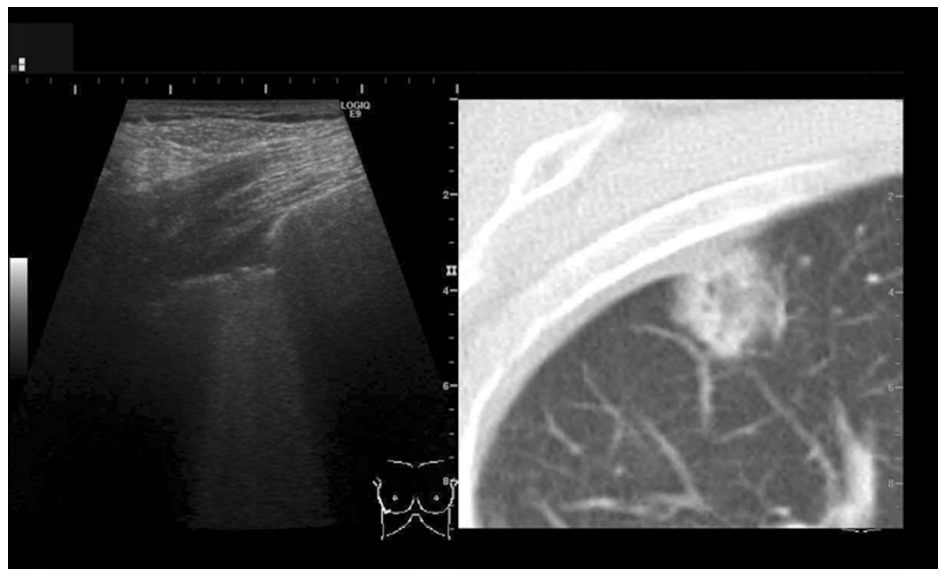

(b) 


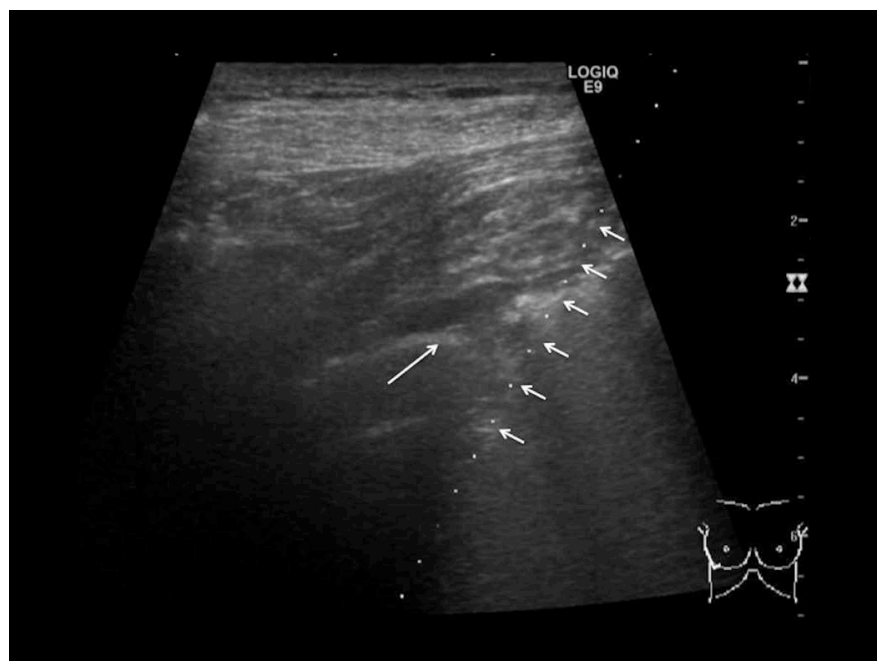

(c)

Figure 2. An 80-year-old man with a 20-mm part-solid GGN. (a) Chest CT scan showed a peripheral pulmonary part-solid GGN; (b) Fusion imaging of the contact area between the pleura and the solid component to localization of the lesion; (c) The inserted line was visualized as a dotted line through the contact area between the pleura and the solid component (long arrow). The biopsy needle (short arrows) was introduced into the lesion along the dotted line; subsequently, two samples were obtained without occurrence of pneumothorax. The biopsy revealed that the lesion was adenocarcinoma.

the CT and US images were linked on the monitor, examination through the fusion image was conducted. The safe needle path was set to pass the biopsy needle through the pleura adjacent to the solid component of the lesion to reduce the risk of pneumothorax. In this study, there were no cases excluded owing to less secure needle guidelines despite identification of the pleura associated with the solid component of the lesion. The same interventional radiologist performed all biopsies with the help of one of eight radiology fellows. All biopsies were performed using puncture-guided devices with imaging. While the probe was fixed firmly to indicate the lesion, the 18-gauge guiding needle (US-guided puncture needle; CREATE MEDIC CO., LTD, Yokohama, Japan) was inserted into the chest wall under local anesthesia with 1\% lidocaine (Xylocaine; Astra-Zeneca, Osaka, Japan). After a 20-gauge automatic cutting needle with a length-throw of 20 mm (Bard Magnum; Bard Biopsy Systems, Tempe, AZ, USA) was introduced through the guiding needle, the patients were told to hold their breath. Under breath-holding, the tissue was punctured using the cutting needle and the needle was then withdrawn from the body. The specimen was immediately placed in an individual formalin-filled container. Acquisition of two specimens is the standard procedure. If pneumothorax occurred immediately after the first puncture, only one sample was obtained. On exposure of the guiding needle to room air during withdrawal of the biopsy needle, the radiologist covered the hole of the guiding needle with his finger to prevent air from flowing into the lung or the pleural cavity. When the operators considered the quantity of the specimen insufficient through visual examination, acquisition of specimens was repeated 
because no pathologist was on site. If the patients complained of symptoms associated with pneumothorax or hemoptysis after the procedure, a chest CT was obtained. In addition, posteroanterior chest radiographs of all patients were obtained on the following day.

\subsection{Data Collection and Study Definition}

One interventional fellowship-trained radiologist (with experience of $>10$ years) evaluated medical records for consecutive patients presenting for US-guided biopsies and assessed the lesions' characteristics and procedure parameters. One radiologist (with experience of $>30$ years) and the same interventional fellowship-trained radiologist independently measured the longest diameters of all lesions on CT. The longest diameter was measured along the long axis of the lesion on the lung window setting of CT. The lesion-pleura contact arc length (LPCAL) of all lesions was measured on CT twice on different days by the same interventional fellowship-trained radiologist. The pleural lesion contact area was defined as a cross-sectional view of the peripheral lung lesion in contact with the overlying pleural surface in the CT mediastinum setting. The LPCAL was measured as a straight-line approximation, in order to obtain the total value of each value $(<10 \mathrm{~mm})$ separately measured along the maximum curvature length of the lesion on the mediastinal window setting of CT. The mean values were accepted for all quantitative data. The lesions were divided into two groups: group 1 (identification on B-mode) and group 2 (identification on fusion image).

The pathological results of the specimens were classified as malignant, benign, and non-diagnostic. Insufficient specimens with a paucity of suspicious cellularity were considered non-diagnostic. For statistical analysis, the pathologically malignant results were regarded as positive in biopsy, while the pathologically benign results were considered as negative in biopsy. The diagnostic accuracy of US-guided biopsy was calculated using the follow formula: diagnostic accuracy $(\%)=$ number of lesions accurately diagnosed (true positive + true negative)/total number of lesions. A true-positive case included a malignant biopsy result confirmed by the surgical specimen or the post-procedural malignant clinical course. A true-negative case included a benign biopsy result together with surgical confirmation or the subsequent clinical course showing no increase in size or the disappearance of lesion with or without administration of antibiotics on follow-up CT for at least 12 months. A case was considered as a false negative when a benign biopsy result was contradicted by the finding of malignancy at surgical resection, biopsy of other organs, or tumor growth observed at follow-up CT examination (performed 3 months after biopsy). Tumor growth was defined according to the Response Evaluation Criteria in Solid Tumors (RECIST). Final diagnosis was defined as a clinical diagnosis at the end of the follow-up period or by September, 2017.

\subsection{Statistical Analyses}

Continuous variables were expressed as the mean and standard deviation. Be- 
tween the two groups, the Mann-Whitney's U test was used to compare quantitative variables, and the chi-square test was used to compare qualitative variables. A $p$ value $<0.05$ was considered to indicate a statistically significant difference. Data processing and analysis were performed with commercially available software (MedCalc version 16.8.4; MedCalc Software, Ostend, Belgium).

\subsection{Results}

Of 50 peripheral lesions, 40 lesions $(80 \%)$ were detected by means of B-mode alone (group 1), and 10 lesions (20\%) were identified by means of fusion image (group 2). In this study, no patient was noted as a registration error in the image fusion approach. The patients' and lesions' characteristics, and procedure parameters between the two groups are shown in Table 1 . Nodule type $(p<0.001)$ and size $(p=0.02)$ of the lesions showed significant differences between the two groups, whereas the other values showed no significant differences. Although pneumothorax occurred immediately after the first puncture in five of $50(10 \%)$ lesions, no symptoms developed in all patients; and the sizes of the five lesions ranged from $4 \mathrm{~mm}$ to $20 \mathrm{~mm}$. These five patients had sufficient tissues available for pathological diagnosis of malignancy. The final diagnosis is summarized in Table 2. As malignant lesions, 36 lesions of primary lung cancer (non-small cell lung cancer/small cell lung cancer $=31 / 5), 5$ lesions of lung metastasis from other organs, 1 lesions of malignant mesothelioma were confirmed. And the remaining 8 lesions were benign lesions (epithelioid cell granuloma/nonspecific $=$ $2 / 6)$. The definitive diagnoses in group 1 were confirmed by surgery $(n=11)$ or clinical course $(\mathrm{n}=29)$, and 10 lesions in group 2 were confirmed by surgery ( $\mathrm{n}$ $=4)$ or clinical course $(n=6)$. Diagnostic accuracy between the two groups is shown in Table 3. The diagnostic accuracy of group 1 and group 2 were $90 \%$ and $100 \%$, respectively. Four lesions with diagnostic failure (three pathological benign lesions and one non-diagnostic lesion) are summarized in Table 4. Of these, malignancy was confirmed in two lesions by lobectomy (No. 1 and No. 3), in one lesion by repeat US-guided lung biopsy (No. 2), and in one lesion by US-guided subclavicular lymph node biopsy (No. 4). With regard to the relationship between lesion-size and LPCAL, the diagnostic accuracy for malignant and benign lesions is summarized in Table 5. All lesions' characteristics using fusion image are shown in Table 6. In this study, life-threatening major complications and hemoptysis were not observed.

Table 1. Patients' and lesions' characteristics and procedure parameters between the two groups.

\begin{tabular}{lrccc}
\hline & & Group 1 & Group 2 & $p$ value \\
\hline \multirow{3}{*}{ Sex } & Patients' characteristics & & & \\
& male & 32 & 8 & 1 \\
& female & 8 & 2 & \\
Age & median & 73 & 78 & 0.64 \\
\hline
\end{tabular}




\section{Continued}

\begin{tabular}{|c|c|c|c|c|}
\hline & range & $50-86$ & $61-85$ & \\
\hline \multicolumn{5}{|c|}{ Lesions' characteristics } \\
\hline \multirow[t]{2}{*}{ Nodule type } & solid & 40 & 7 & $<0.001$ \\
\hline & part-solid GGN & 0 & 3 & \\
\hline \multirow[t]{2}{*}{ Size $(\mathrm{mm})$} & median & 29 & 17 & 0.02 \\
\hline & range & $9.5-116$ & $4-38$ & \\
\hline \multirow[t]{2}{*}{ LPCAL (mm) } & median & 23.8 & 15.6 & 0.09 \\
\hline & range & $6.1-162.7$ & $2.8-29.5$ & \\
\hline \multirow[t]{10}{*}{ Location } & right upper lobe & 9 & 2 & 0.87 \\
\hline & (S1/S2/S3/multiple) & $(3 / 4 / 1 / 1)$ & $(1 / 0 / 1 / 0)$ & \\
\hline & right middle lobe & 0 & 1 & 0.05 \\
\hline & (S4/S5/multiple) & $(0 / 0 / 0)$ & $(1 / 0 / 0)$ & \\
\hline & right lower lobe & 14 & 3 & 0.77 \\
\hline & (S6/S7/S8/S9/S10/multiple) & $(2 / 0 / 2 / 2 / 7 / 1)$ & $(2 / 0 / 0 / 1 / 0 / 0)$ & \\
\hline & left upper lobe & 12 & 3 & 1 \\
\hline & (S1 + 2/S3/S4/S5/multiple) & $(9 / 1 / 1 / 1 / 0)$ & $(3 / 0 / 0 / 0 / 0)$ & \\
\hline & left lower lobe & 5 & 1 & 0.83 \\
\hline & (S6/S8/S9/S10/multiple) & $(1 / 0 / 0 / 3 / 1)$ & $(1 / 0 / 0 / 0 / 0)$ & \\
\hline \multicolumn{5}{|c|}{ Procedure parameters } \\
\hline \multirow[t]{3}{*}{ Patient position } & supine & 19 & 5 & 0.89 \\
\hline & prone & 12 & 1 & 0.2 \\
\hline & lateral & 9 & 4 & 0.26 \\
\hline \multirow[t]{2}{*}{ No. of puncture } & median & 2 & 2 & 0.36 \\
\hline & $(1 / 2 / 3 / 4)$ & $(4 / 25 / 9 / 2)$ & $(1 / 8 / 1 / 0)$ & \\
\hline \multirow{2}{*}{$\begin{array}{c}\text { Pnx }{ }^{*} \text { after first } \\
\text { puncture }\end{array}$} & present & 4 & 1 & 0.39 \\
\hline & absent & 36 & 9 & \\
\hline \multirow[t]{2}{*}{ Hemoptysis } & present & 0 & 0 & - \\
\hline & absent & 40 & 10 & \\
\hline
\end{tabular}

$\mathrm{Pnx}^{*}$ : Pneumothorax.

Table 2. Final diagnosis between the two groups.

\begin{tabular}{|c|c|c|c|}
\hline & & Group 1 & Group 2 \\
\hline \multicolumn{4}{|l|}{ Malignant } \\
\hline & adenocarcinoma & 19 & 6 \\
\hline & squamous cell carcinoma & 5 & 1 \\
\hline & small cell carcinoma & 5 & 0 \\
\hline & malignant mesothelioma & 0 & 1 \\
\hline & metastases & 4 & 1 \\
\hline \multicolumn{4}{|l|}{ Benign } \\
\hline & epithelioid cell granuloma & 2 & 0 \\
\hline & non-specific benign & 5 & 1 \\
\hline
\end{tabular}


Table 3. Diagnostic accuracy between the two groups.

\begin{tabular}{ccc}
\hline & Group 1 & Group 2 \\
\hline True positive & 29 & 9 \\
True negative & 7 & 1 \\
False negative & 3 & 0 \\
Non-diagnostic for malignancy & 1 & 0 \\
Diagnostic accuracy & $90 \%$ & $100 \%$ \\
\hline
\end{tabular}

Table 4. Results of lesions with diagnostic failure.

\begin{tabular}{|c|c|c|c|c|c|c|}
\hline No & type & Size & LPCAL & $\mathrm{Pnx}^{*}$ & Pathological diagnosis & Final diagnosis \\
\hline \multicolumn{7}{|c|}{ False negative } \\
\hline 1 & solid & 42 & 16.4 & $(-)$ & lymphoid infiltration & adenocarcinoma \\
\hline 2 & solid & 25 & 17 & $(-)$ & fibrous tissue & metastasis \\
\hline 3 & solid & 18.3 & 16.7 & $(-)$ & inflammation & adenocarcinoma \\
\hline \multicolumn{7}{|c|}{ Non-diagnostic for malignancy } \\
\hline 4 & solid & 41.1 & 39.4 & $(-)$ & $\begin{array}{l}\text { necrotic tissue with } \\
\text { a few malignant cells }\end{array}$ & squamous cell carcinoma \\
\hline
\end{tabular}

Pnx*: Pneumothorax

Table 5. Results of biopsies according to size and LPCAL between the two groups.

\begin{tabular}{ccccccccccccc}
\hline & \multicolumn{1}{c}{ Group 1 } & \multicolumn{7}{c}{ Group 2 } \\
\hline Malignant & \multicolumn{3}{c}{ Size } & \multicolumn{3}{c}{ LPCAL } & & Size & \multicolumn{3}{c}{ LPCAL } \\
\hline (mm) & TP & FN & ND & TP & FN & ND & TP & FN & ND & TP & FN & ND \\
\hline 10 & & & & 4 & & & 1 & & & 2 & & \\
$11-20$ & 9 & 1 & & 7 & 3 & & 7 & & & 4 & & \\
$21-30$ & 6 & 1 & & 7 & & & & & & 3 & & \\
$31-40$ & 6 & & & 2 & & 1 & 1 & & & & & \\
$>40$ & 8 & 1 & 1 & 9 & & & & & & & & \\
Total & 29 & 3 & 1 & 29 & 3 & 1 & 9 & 0 & 0 & 9 & 0 & 0 \\
\hline Benign & SB & NB & ND & SB & NB & ND & SB & NB & ND & SB & NB & ND \\
\hline 10 & 1 & & & 1 & & & & & & & & \\
$11-20$ & & 3 & & & 1 & & & 1 & & & & \\
$21-30$ & & & & & 1 & & & & & & 1 & \\
$31-40$ & 1 & 1 & & & 1 & & & & & & & \\
$>40$ & & 1 & & 1 & 2 & & & & & & & \\
Total & 2 & 5 & 0 & 2 & 5 & 0 & 0 & 1 & 0 & 0 & 1 & 0 \\
\hline
\end{tabular}

TP: true positive, FN: false negative, ND: non-diagnostic, SB: specific benign, NB: non-specific benign.

Table 6. Results of biopsy lesions using fusion image.

\begin{tabular}{lccccl}
\hline No & Type & Size & LPCAL & Pnx $^{*}$ & Final diagnosis \\
\hline \multicolumn{2}{l}{ True positive } & & & & \\
1 & solid & 20 & 14.3 & $(-)$ & adenocarcinoma \\
\hline
\end{tabular}




\begin{tabular}{cccccc}
\multicolumn{2}{l}{ Continued } & \multicolumn{1}{l}{} \\
\hline 2 & part-solid GGN & 17 & 14.7 & $(-)$ & adenocarcinoma \\
3 & solid & 4 & 2.8 & $(+)$ & metastasis \\
4 & solid & 17 & 26.9 & $(-)$ & squamous cell carcinoma \\
5 & solid & 18.5 & 22.5 & $(-)$ & malignant mesothelioma \\
6 & part-solid GGN & 38 & 12.2 & $(-)$ & adenocarcinoma \\
7 & solid & 12 & 16.4 & $(-)$ & adenocarcinoma \\
8 & part-solid GGN & 15 & 9.7 & $(-)$ & adenocarcinoma \\
9 & solid & 19.7 & 29.5 & $(-)$ & adenocarcinoma \\
True negative & & & & \\
10 & solid & 15.5 & 21.9 & $(-)$ & no malignancy \\
\hline
\end{tabular}

Pnx*: Pneumothorax.

\section{Discussion}

Characteristics of the target lesions and procedure parameters may affect B-mode guided biopsy procedure accuracy. Liao et al. reported that the correct diagnosis of lesions ( $\leq 30 \mathrm{~mm}$ in diameter) was obtained in 48 of 50 (96\%) peripheral thoracic lesions [6]. Previous reports of US-guided thoracic biopsy indicated that lesion-size did not influence the diagnostic accuracy [4] [6] [8] [9]. However, lesions that were not detected in the conventional B-mode alone may have been excluded in these reports. In this study, 10 of 50 lesions were detected using the fusion image, and the diagnostic accuracy of $9(<30 \mathrm{~mm}$ in size) of 10 lesions was $100 \%$ (9/9 lesions). Although a predictive statistical comparison of the diagnostic accuracy of the biopsy between the B-mode alone and the fusion image was not conducted in this study, the fusion image might be able to improve diagnostic accuracy and clarify a relationship between yield and minimal lesion size.

Jeon et al. reported that the diagnostic accuracy of US-guided biopsy using B-mode was significantly increased for lesions in which the pleural-lesion contact area was $\geq 30 \mathrm{~mm}$ on the CT images [8]; moreover, for lesions with LPCAL values $\leq 30 \mathrm{~mm}$, the diagnostic accuracy decreased from $98 \%$ to $85.4 \%$. They concluded that US-guided biopsy of pulmonary lesions might be performed more easily when the lesion has a contact area as wide as the US window, which permits a flexible approach route. In this study, LPCAL showed no statistically significant difference between the two groups $(p=0.09)$. However, the LPCAL of 10 lesions using fusion image was $<30 \mathrm{~mm}$ and the diagnostic accuracy was $100 \%$ (10/10 lesions). On the other hand, the diagnostic accuracy of biopsy using B-mode alone decreased from $93.8 \%$ (15/16 lesions) to $87.5 \%$ (21/24 lesions) by using an LPCAL of $30 \mathrm{~mm}$ as the boundary criteria. For good sampling of US-guided biopsy of the lung, it is important that the needle tip passes exactly through the contact area. A false-positive contact area may be detected by B-mode alone, even if the lesion conspicuity is considered sufficient for biopsy. Diagnostic accuracy guided with fusion images may have a higher diagnostic 
yield for lesions with LPCAL $<30 \mathrm{~mm}$, although a larger prospective study is needed.

In this study, no life-threatening symptoms were observed. Although pneumothorax occurred immediately after the first puncture in five of $50(10 \%)$ lesions, a chest tube was not placed due to the patients' asymptomatic conditions. In previous reports related to transthoracic biopsy of peripheral thoracic lesions through US, the frequency of pneumothorax ranged from $2.6 \%$ to $4 \%$, and the rate of chest tube drainage ranged from $0 \%$ to $1 \%$ [6] [16] [17]. Moreover, hemoptysis was not observed during all procedures. Previous reports have indicated that hemoptysis associated with US-guided lung biopsy is an uncommon complication [6] [16]. Thus, hemoptysis might rarely be a life-threatening condition in patients undergoing biopsy under US guidance.

This study had some limitations. First, it was a retrospective study and included a small number of lesions using fusion image. Second, the final diagnosis was confirmed in surgically resected specimens obtained from only 15 lesions. Third, the choice to use fusion image was made by the same operator who performed the procedure. Because the selection and management for a US-guided lung biopsy depends on the practice of the individual using US, lesion characteristics can be altered by each operator. Finally, the possibility of asymptomatic patients with pneumothorax and bleeding associated with US-guided biopsy was not investigated.

\section{Conclusion}

Fusion images created using real-time US and CT may be useful for identification of the minimal size of potential target lung lesions and may be more suitable for improved yields with US-guided lung biopsy.

\section{Conflicts of Interest}

The authors declare no conflicts of interest regarding the publication of this paper.

\section{References}

[1] Sconfienza, L.M., Mauri, G., Grossi, F., et al. (2013) Pleural and Peripheral Lung Lesions: Comparison of US- and CT-Guided Biopsy. Radiology, 266, 930-935. https://doi.org/10.1148/radiol.12112077

[2] Jarmakani, M., Duguay, S., Rust, K., et al. (2016) Ultrasound versus Computed Tomographic Guidance for Percutaneous Biopsy of Chest Lesions. Journal of Ultrasound in Medicine, 35, 1865-1872. https://doi.org/10.7863/ultra.15.10029

[3] Lee, M.H., Lubner, M.G., Hinshaw, J.L., et al. (2018) Ultrasound Guidance versus CT Guidance for Peripheral Lung Biopsy: Performance According to Lesion Size and Pleural Contact. American Journal of Roentgenology, 210, W110-W117. https://doi.org/10.2214/AJR.17.18014

[4] Yang, P.C., Lee, Y.C., Yu, C.J., et al. (1992) Ultrasonographically Guided Biopsy of Thoracic Tumors. A Comparison of Large-Bore Cutting Biopsy with Fine-Needle Aspiration. Cancer, 69, 2553-2560. 
https://doi.org/10.1002/1097-0142(19920515)69:10<2553::AID-CNCR2820691027> 3.0.CO;2-4

[5] Sheth, S., Hamper, U.M., Stanley, D.B., et al. (1999) US Guidance for Thoracic Biopsy: A Valuable Alternative to CT. Radiology, 210, 721-726. https://doi.org/10.1148/radiology.210.3.r99mr23721

[6] Liao, W.Y., Chen, M.Z., Chang, Y.L., et al. (2000) US-Guided Transthoracic Cutting biopsy for Peripheral Thoracic Lesions Less Than $3 \mathrm{~cm}$ in Diameter. Radiology, 217, 685-691. https://doi.org/10.1148/radiology.217.3.r00dc21685

[7] Diacon, A.H., Schuurmans, M.M., Theron, J., et al. (2004) Safety and Yield of U1trasound-Assisted Transthoracic Biopsy Performed by Pulmonologists. Respiration, 71, 519-522. https://doi.org/10.1159/000080638

[8] Jeon, K.N., Bae, K., Park, M.J., et al. (2014) US-Guided Transthoracic Biopsy of Peripheral Lung Lesions: Pleural Contact Length Influences Diagnostic Yield. Acta Radiologica, 55, 295-301. https://doi.org/10.1177/0284185113494984

[9] Yuan, A., Yang, P.C., Chang, D.B., et al. (1992) Ultrasound-Guided Aspiration Biopsy of Small Peripheral Pulmonary Nodules. Chest, 101, 926-930. https://doi.org/10.1378/chest.101.4.926

[10] Meena, N. and Bartter, T. (2015) Ultrasound-Guided Percutaneous Needle Aspiration by Pulmonologists: A Study of Factors with Impact on Procedural Yield and Complications. Journal of Bronchology \& Interventional Pulmonology, 22, 204-208.

[11] Rowan, K.R., Kirkpatrick, A.W., Liu, D., et al. (2002) Traumatic Pneumothorax Detection with Thoracic US: Correlation with Chest Radiography and CT-Initial Experience. Radiology, 225, 210-214. https://doi.org/10.1148/radiol.2251011102

[12] Reissig, A. and Kroegel, C. (2005) Accuracy of Transthoracic Sonography in Excluding Post-Interventional Pneumothorax and Hydropneumothorax. Comparison to Chest Radiography. European Journal of Radiology, 53, 463-470. https://doi.org/10.1016/j.ejrad.2004.04.014

[13] Lee, M.W. (2014) Fusion Imaging of Real-Time Ultrasonography with CT or MRI for Hepatic Intervention. Ultrasonography, 33, 227-239. https://doi.org/10.14366/usg.14021

[14] Yamamoto, S., Maeda, N., Tamesa, M., et al. (2012) Prospective Ultrasonographic Prediction of Sentinel Lymph Node Metastasis by Real-Time Virtual Sonography Constructed with Three-Dimensional Computed Tomography-Lymphography in Breast Cancer Patients. Breast Cancer, 19, 77-82.

https://doi.org/10.1007/s12282-011-0275-4

[15] Dong, Y., Mao, F., Wang, W.P., et al. (2015) Value of Contrast-Enhanced Ultrasound in Guidance of Percutaneous Biopsy in Peripheral Pulmonary Lesions. BioMed Research International, 2015, Article ID: 531507. https://doi.org/10.1155/2015/531507

[16] Mathis, G., Bitschnau, R., Gehmacher, O., et al. (1999) Ultrasound-Guided Transthoracic Puncture. Ultraschall in der Medizin, 20, 226-235.

[17] Sartori, S., Tombesi, P., Trevisani, L., et al. (2007) Accuracy of Transthoracic Sonography in Detection of Pneumothorax after Sonographically Guided Lung Biopsy: Prospective Comparison with Chest Radiography. American Journal of Roentgenology, 188, 37-41. https://doi.org/10.2214/AJR.05.1716 